\title{
Drivers' emotional evaluation in the noisy environment of the control cabin of a shield tunneling machine
}

\author{
Liangbin Zhang ${ }^{1,2, a}$, Hanbin Luo, b \\ ${ }^{1}$ Department of Construction Management, School of Civil Engineering \& Mechanics, Huazhong \\ University of Science \& Technology, Wuhan, Hubei, China \\ ${ }^{2}$ Wuhan Polytechnic, Wuhan, Hubei, China \\ azinner@163.com, ’lhblhb1963@vip.sina.com
}

\begin{abstract}
Keywords: Emotional evaluation, Noisy environment, Control cabin of shield tunneling machine. Abstract. Being a driver in the control cabin of a shield tunneling machine is a high-pressure career. The mood swings of a driver are vital to his occupational health and construction safety; however, a driver's emotional intensity in a noisy environment have not been considered. This study aims to investigate how a driver's emotional intensity in a noisy environment can be altered. On-site measurements were conducted in an urban metro system, and an emotional survey was performed. Results indicate that the driver's emotion is influenced when the sound pressure level increases to $94.5 \mathrm{dBA}$. The relationship was significant between the emotional intensity and the sound pressure level. The fear was highly evident with the sound pressure level increase given that the drivers were concerned about operational errors. The disliking influenced the heart rate more obviously than the other two emotional types. The driver's emotion has a relationship with social background.
\end{abstract}

\section{Introduction}

Noise is undesired sound, which is a pollutant that affects human health [1]. The health effects of noise can be divided into two categories, namely, auditory health effects and non-auditory health effects [1]. In terms of auditory health effects, hearing loss is a significant health effect [2]. In a construction site, construction noise is the most common source of noise pollution, which can cause several risks to workers' health and safety [3]. non-auditory health effects include annoyance [4], cardiovascular disease [5], cognitive performance [6], human behaviors [7], and sleep disturbance [8]. In recent years, researchers have paid considerable attention to non-auditory health effects [1].

With the growth of urban population, the capacity of public transports and road surfaces is unable to meet the requirement of human transportation. Metro systems had been considered as an important factor to improve the quality of transportation and relieve congestion by filling the gaps of insufficient public transports and road surfaces capacity [9]. In the construction of metro systems, a shield tunneling machine is the most common machinery used for constructing metro lines. The quality and safety of tunnel constructions were considered to achieve the important results of the construction progress of metro systems. The status of a shield tunneling machine would display on the operational screen in the control cabin of the shield tunneling machine, and the drivers must observe the change in the status of the factors continuously. Thus, the driver of the control cabin of the shield tunneling machine is a key artificial factor to ensure the safety and quality of metro constructions, and being a driver is a high-pressure underground work in metro constructions. The subjective reports had indicated that underground workers were less satisfied with their surroundings given that their environment hindered their work and that their emotions were extremely anxious, depressed, and hostile [10]. However, the researches confirm less investigation about the relationship between the emotions of a shield tunneling machine driver and the noisy environment.

This study therefore aims to investigate a shield tunneling machine driver's emotional evaluation in a noisy environment. The research questions in this paper include three aspects: 1 , determine a driver's overall evaluation of a noisy environment. 2, investigate the relationship between sound pressure level in the control cabin of the shield tunneling machine and the drivers' emotional evaluation. 3, determine the effects of a driver's social background on a driver's emotional evaluation 
in a noisy environment. A typical Wuhan metro line was chosen as the case site, and the noise level measurements and a self-administered questionnaire survey were used for data collection.

\section{Methodology}

The development of Chinese metro systems is currently boosting, reaching a historically high level in terms of construction speed, scale, and investment [11]. Wuhan metro lines, which are under construction through the shield tunneling machine, were chosen at the case site. There are 60-70 shield tunneling machines working for Wuhan metro lines on a daily basis. Therefore, enough shield tunneling machine drivers as subjects were available for this study.

The measurement of the noise level was conducted immediately when each subject began to fill out the questionnaire. In this study, a class I sound level meter was used for the data acquisition, measuring the sound pressure level (SPL). The place of measurement was in the shield tunneling machine's control cabin. During the measurement, the microphone of the sound level meter was positioned over $1 \mathrm{~m}$ away from any reflective surface and $1.2-1.5 \mathrm{~m}$ above the floor. The sound level meter was set in slow mode and A-weighting, and reading was acquired every 3-5 second. A total of 5 minute data were obtained in each shield tunneling machine's control cabin, which was used for identifying relationships with emotional factors.

Emotions are pervasive, an inseparable part of the human experience and life. Emotions shape perceptions and direct behavior and influence interactions with others [12]. Before the formal questionnaire was formatted, this paper took a test to indicate whether the drivers' emotion were influenced by the noisy environment in the control cabin as one influencing factor, a correlations analysis was used. The result exhibited that there was a significant correlation between the hearing protection worn and drivers' emotional responses (2-tailed significant value is $0.025, \mathrm{p}<0.05$ ). Subsequently, the current study chose three words of negative emotions: anxiety, fear, and disliking. These three words served as the different types of emotional evaluation of the shield tunneling machine driver in the emotion survey as discussed by the psychologists, the managers of the shield tunneling machine, and the drivers. In terms of the evaluation of the emotions, a five-point emotional intensity scale was used in the questionnaire design. The evaluation of emotion intensity was divided into five levels: $1=$ never, $2=$ rarely, $3=$ sometimes, $4=$ most of the time, and $5=$ always.

In this study, factor analysis was performed on the emotional evaluation item results from the questionnaire, resulting in a KMO (Keiser-Meyer-Olkin) $=0.735$, Cronbach's $\alpha$ is 0.723 , which were within an acceptable range.

A total of 112 shield tunneling machine drivers took part in the experiment. The subjects in all the field surveys were randomly selected, and their educational and social backgrounds were proven to be representative [13]. Given that the shield tunneling machine drivers' social background may also influence their evaluation of emotion, the subjects were sorted into four categories in terms of age: $\leq 24,25-30,31-35$, and $\geq 36$ years old; three categories in terms of educational levels: senior middle school, technical school, and college graduates; and four categories in terms of years of service: $\leq 2$, $3-5,6-8, \geq 9$ years. Given that the subjects need time, approximately 20-30 minutes to accommodate the sound environment in the spaces [14], the drivers who were in the shield tunneling machine's control cabin for less than half an hour were not interviewed.

\section{Results and disscussion}

Several graphs were presented to show the emotion evaluation results. Fig. 1 shows different emotions corresponding to the range of SPL, where it can be seen that the shield tunneling machine drivers were more likely to reflect the anxiety emotion than the other two emotional types when the value of the SPL was between 92 and $100 \mathrm{dBA}$. However, the fear and disliking emotions of the drivers tended to take place in a scope from 96 to $102 \mathrm{dBA}$ and from 96 to $100 \mathrm{dBA}$, respectively. 


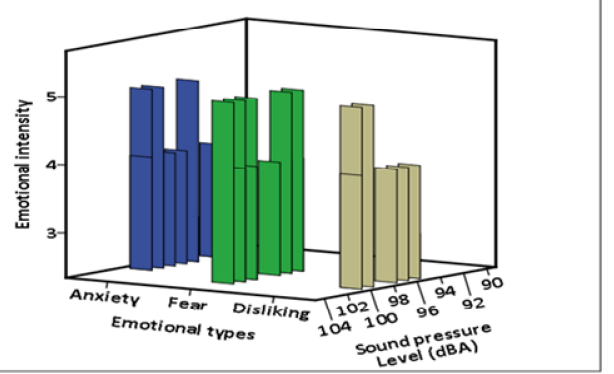

Fig. 1. Different emotion corresponding to range of SPL.

Fig. 2 shows the relationship between the degree of emotion on SPL in the control cabin and the measured SPL synchronous with the questionnaire. The degree of emotion evaluation was expressed by mean of emotional intensity, which corresponded to the mean of "anxiety", "fear" and "disliking" results. As the mean value of SPL increased, the degree of emotion evaluation showed an upward trend. The correlation coefficient between them was $\mathrm{r}=0.7(\mathrm{p}<0.01)$. The average SPL value corresponded to approximately $93.6 \mathrm{dBA}$ when the emotion evaluation was "Rarely." When the emotion evaluation was "Sometimes", the average SPL value was 94.5 dBA. When the emotion evaluation was "Most of time", the average SPL value was $97.8 \mathrm{dBA}$. The average SPL value was 99.1 dBA when the emotion evaluation was "Always." Producing a higher emotional intensity would be easy when an SPL value exceeded $98 \mathrm{dBA}$ in the control cabin. Evidently, the noise factor

influenced the emotion.

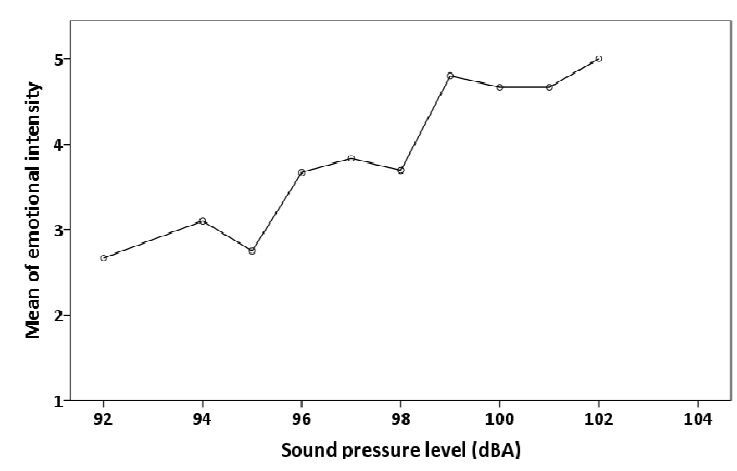

Fig. 2. Mean of emotional intensity as a function of SPL.

Notably a general correlation between the SPL and the three emotional types exists $(\mathrm{p}<0.001)$. Fig. 3 shows the relationship between emotional intensity and SPL of the drivers' different emotional types. It can be seen that the drivers' emotional intensity of the anxiety, fear, and disliking emotion increased with SPL increased. The coefficients of determination R2 was $0.49,0.534$, and 0.805 , respectively. This result confirmed that the rate of the emotional intensity increase in the fear emotion was the fastest among the three emotional intensities. According to the interview, some drivers said, "Driver is a high-pressure career." Other drivers said, "The environment of the control cabin is noisy, this environment makes me fearful most of the time." Some drivers also said, "The operational error is the biggest concern." Hence, the reason was that the drivers were concerned about operational errors, and the fear emotion was highly significant as the SPL increased in the noisy environment.

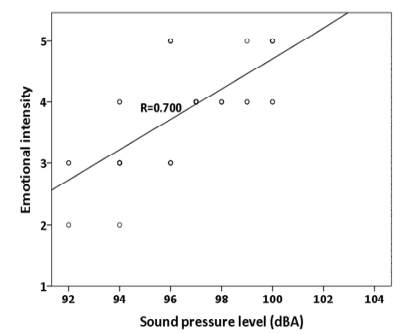

(a)

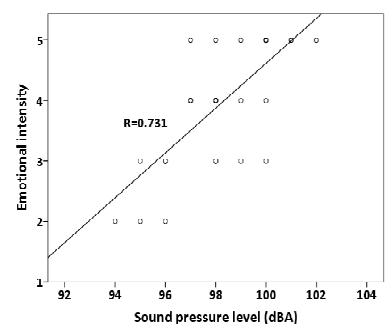

(b)

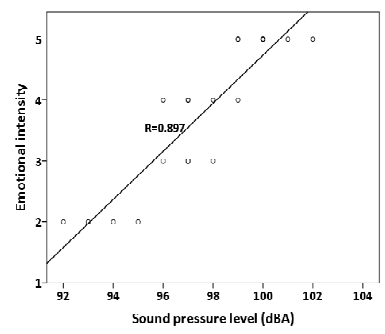

(c)

Fig. 3. Relationship between emotional intensity and SPL of the drivers' different emotional types: (a) anxiety; (b) fear; and (c) disliking. 
The difference in the drivers' emotional intensity was determined through their social background for every emotional type. The results of the relationship between emotional intensity and social background of drivers are shown in Table 1 . The age difference was significant $(\mathrm{p}<0.05)$ with the correlation coefficient ranging from 0.16 to 0.26 . For anxiety and fear emotions, emotional intensity was higher when the drivers were younger, except for the disliking emotion. The reason may be that the drivers' work experience was insufficient when their age was less, the drivers showed anxiety and fear emotions in the noisy environment. However, with the increase in age and enough work experience of the drivers, the drivers showed a higher disliking. Interestingly, the difference in the educational level and years of service was also significant $(p<0.05)$ in the drivers' emotional intensity, with the correlation coefficient ranging from 0.34 to 0.51 for the educational level and 0.24 to 0.44 for years of service. Evidently, the drivers' emotion showed a higher disliking with a high educational level or a long service. The result proves that the drivers who had strong cognitive ability and long years of service easily show the disliking emotion in a noisy environment.

Table 1 Pearson correlation coefficient (two-tailed) between emotional intensity and social background of drivers.

\begin{tabular}{l|lll}
\hline & ANXIETY & FEAR & DISLIKING \\
\hline Age & $-0.21^{*}$ & $-0.16^{*}$ & $0.26^{*}$ \\
Educational level & $0.34^{*}$ & $0.37^{*}$ & $0.51^{*}$ \\
Service year & $0.24^{*}$ & $0.31^{*}$ & $0.44^{*}$
\end{tabular}

\section{Conclusions}

The drivers' emotions were influenced by the average sound pressure level value in the control cabin when it increased to $94.5 \mathrm{dBA}$. The emotional intensity was increased with increased sound pressure level. Fear was highly evident as sound pressure level increased due to the drivers' concern about operational errors.

The drivers' emotion had a significant relationship with the social background. With a high educational level, increased age and long years of service, showed a higher disliking emotion.

On the basis of this research, the conclusions can be regarded as a pattern to ensure the safety and quality of metro constructions through controlling drivers' emotion in the noisy environment of the control cabin of a shield tunneling machine. Future research is required to further investigate the long-term effects of a noisy environment on the emotional evaluation based on drivers' operational procedures for a shield tunneling machine.

\section{References}

[1] Basner M, Babisch W, Davis A, Brink M, Clark C, Janssen S, et al: submitted to Journal of Lancet (2014).

[2] Karpa MJ, Gopinath B, Beath K, Rochtchina E, Cumming RG, Wang JJ, et al: submitted to Journal of Ann Epidemiol (2010).

[3] Lee SC, Hong JY, Jeon JY: submitted to Journal of Building and Environment (2015).

[4] Janssen SA, Vos H, Eisses AR, Pedersen E: submitted to Journal of J Acoust Soc Am (2011).

[5] Haralabidis AS, Dimakopoulou K, Vigna-Taglianti F, Giampaolo M, Borgini A, Dudley ML, et al: submitted to Journal of Eur Heart J (2008).

[6] Hygge S, Evans GW, Bullinger M: submitted to Journal of Psychol Sci (2002).

[7] Meng Q, Kang J: submitted to Journal of Science of The Total Environment (2016).

[8] Basner M, Samel A, Isermann U: submitted to Journal of J Acoust Soc Am (2006). 
[9] Vasconcellos EA, in: Urban transport, environment, and equity: the case for developing countries, Earthscan Publishing, London (2001), in press.

[10] D. Hollon S, C. Kendall P, Norsted S, Watson D: submitted to Journal of Underground Space (1980).

[11] Xu J, Ding L: submitted to Journal of Frontiers of Engineering Management (2017).

[12] Frijda NH, in: The Emotions: Cambridge University Press, London (1986), in press.

[13] Yu L, Kang J: submitted to Journal of Applied Acoustics (2010).

[14] Meng Q, Kang J: submitted to Journal of PLOS ONE (2013). 